\title{
Comparison of branded and non-branded food samples widely consumed in north India with reference to Trans fatty acid content
}

\author{
Prashasti Tripathi* \\ Centre of Food Technology, University of Allahabad, Allahabad (Uttar Pradesh), India \\ Vineeta Puranik \\ Centre of Food Technology, University of Allahabad, Allahabad (Uttar Pradesh), India \\ Shalini Purwar \\ Department of Basic and Social Sciences, Banda University of Agriculture and Technology \\ (Banda), India \\ ${ }^{*}$ Corresponding author. Email: prashasti.tripathi@gmail.com
}

\section{Article Info}

https://doi.org/10.31018/

jans.v13i1.2449

Received: December 6, 2020

Revised: January 20, 2021

Accepted: January 27, 2021

\section{How to Cite}

Tripathi, P. et al. (2021). Comparison of branded and non-branded food samples widely consumed in north India with reference to Trans fatty acid content. Journal of Applied and Natural Science, 13(1),16 - 25. https://doi.org/10.31018/jans.v13i1.2449

\begin{abstract}
Trans fatty acids (TFA) are the geometrical isomers of monounsaturated and polyunsaturated fatty acids that affect the functional and physicochemical properties of these fatty acids, which in turn affect their metabolism in humans. Since the database available for trans fatty acids in food from India is scarce, the research report generates data about trans fatty acid content in selected foods popular in north India. In this report, various food samples like cookies, chocolates, biscuits, pizza, fries, indigenous snacks like samosa, pakora and indigenous sweets like jalebi, gulab jamun, and laddoo were analyzed for the Trans Fatty Acid (TFA) content by gas chromatography. A large variation was found in trans fatty acid content among these food samples. The results also showed that only $4.5 \%$ of the samples were found to contain TFA less than $0.5 \%$ while approximately $8 \%$ of samples having more than 5\% TFA ( 1 branded and 6 non-branded samples). Also, a large variation was found in the trans fatty acid content of branded and non-branded food samples with the mean value of TFA in branded and non-branded food groups as 1.781 and 6.125 respectively and the t-value of 0.852 between the two groups. When regulations are emphasizing on labelling the TFA content on the product, there are arrays of unlabelled products which are not governed under any regulations. Hence there is a need for strong food regulations to bring levels of trans fats in processed foods to negligible levels.
\end{abstract}

Keywords: Gas chromatography, Indigenous foods, Labeling, Peroxide value, Trans fatty acid

\section{INTRODUCTION}

In present times a sound and healthy body are becoming our priority. People are consuming immunity boosting foods to combat any kind of infection, but along with the consumption of healthy food, we should also focus on avoiding foods that may adversely affect our health and immune system. These lifestyle diseases typically involve immune dysfunction, leading to increased inflammation and poorer health outcomes. More recently over the 20th century, not only has there been an increase in the rate of fat consumption in individuals but also a shift in the quality of fat consumed, concurrent with industrial food practices (Hosomi et al., 2020). The current study was conducted with the aim to find the intake of bad fat in foods available in the market. Trans fatty acids (TFA) are the geometrical isomers of unsatu- rated fatty acids with at least one non-conjugated, carbon-carbon double bond in the trans configuration rather than the more common cis configuration (Codex, 1985; EFSA, 2004; Kodali, 2005). Trans isomers generally have more density, lower solubility and higher melting point. Often food manufacturers use artificial trans fat in food products because it is inexpensive and it increases the food's shelf life, stability, and texture. The trans configuration has an effect on the functional and physicochemical properties of these fatty acids which in turn affects their metabolism in humans (Mozaffarian et al., 2010). This process maintains the taste and smell characteristics of oils while enabling a longer shelf life for final food products. Trans fat can be found in foods such as vegetable shortenings, margarines, candies, cookies, snack foods, fried foods, crackers, baked goods, and other processed foods prepared 
from partially hydrogenated vegetable oils. After absorption, TFA follow the same metabolic routes as other fatty acids and selective accumulation in tissues does not occur. Ultimately, TFA are oxidized to provide energy. Although there is some evidence from in vitro and animal studies that conversion of essential fatty acids is inhibited by TFA, metabolism of essential fatty acids is unlikely to be impaired by TFA when intakes of essential fatty acids meet recommended levels (Micha and Mozaffarian, 2009). Consumption of Trans fatty acid in diet may lead to various health issues like cardiovascular disease, obesity, coronary heart diseases, prostate cancer, breast cancer, etc. It may also affect the cell membrane and autoimmune system and can cause damage to brain cells (Zhu et al., 2019). Hence it is required to minimize the intake of trans fatty acids, which are not indispensable to humans. World Health Organization has also recommended that trans fats be limited to less than $1 \%$ of overall energy intake (WHO, 2003). In order to implement the regulations for limiting the trans fatty acid content in diet has caused a request for a database on trans fatty content in foods consumed in different countries. Consequently, there is a demand to perform analyses for trans fatty acids in different kind of foods that contain fat components since there is a lack of a database for trans fatty acid level in foods. The increasing complexity of the food supply coupled with variations in the fats and oils used by the food industry and the availability of a wide range of processed and manufactured foods, make the compilation of the fatty acid composition of foods difficult. McNaughton et al. (2007) also reported a difference in the intake of trans fatty acids in two population groups and reported that mean intakes were significantly different between the WFR (weighted food records) and FFQ (selfadministered) for total trans fatty acids, with the FFQ providing higher estimates than the WFR. Also, during cooking, many changes occur that can potentially influence TFA content in food. Lack of a comprehensive database on the trans fatty acid content of food particular to a region is often a hurdle in nutritional studies. Most of the data available from India are not analyzed technically by standard methods but are estimated by the use the food composition and analysis tables of the Indian National Institute of Nutrition (Aggrawal et al., 2008). When there are regulations which emphasize on labelling the TFA content in foods, there are a number of unlabelled, unpacked products also which are not governed under any regulations which include: cakes, pastries, snacks, sweets, namkeens etc. Hence a study was undertaken to assess the trans fatty acid content of widely consumed popular snacks and sweets from North India This study helps to create a healthy food environment that helps in the acceptability of healthier food, improved nutrition labelling, restricting food advertising and can make better support to consumers to select healthier food choices.

\section{MATERIALS AND METHODS}

For the purpose of analysis of trans fat intake of food items, sixty four different food samples widely consumed like different bakery products, such as biscuits, cookies and cakes, Indian traditional sweets, traditional snacks, etc. were included for study as these products are widely consumed and popular among the locals. The samples for determination of Trans fatty acids were procured from the fast-food restaurants, street foods vendors and packed foods available in the market from Allahabad (covering all major market places like civil lines, katra, chowk, allahpur etc.). The details of all the selected samples had been submitted to the Centre of Food Technology, University of Allahabad where the analysis was performed in 2017. For trans fat estimation 200-500 gm of sample was collected depending upon the total fat content of the food product.

Total samples were categorized into six food groups viz. fat/oil, baked products, confectionery, snacks, indigenous sweets and indigenous snacks. The peroxide value and free fatty acid content of samples were also calculated and the results were compared by dividing/ separating the samples into two groups of branded and non-branded products.

\section{Reagents and standards}

All chemicals, solvents and reagents employed were of analytical grade and purchased from Merck (India). The internal standard (IS) pentadecanoic acid (C15:0) and the individual of five Fatty acids [FA] and Fatty acid methyl Esters [FAME] standards: Palmitelaidic acid (PA) C16:0, Elaidic acid (EA) C18:1t9, Vaccenic acid (VA) (C18:1t11), Linoleic acid isomer mix (LA) C18:2, and Linolenic acid isomer mix (LLA) C18:3, were purchased from Sigma-Aldrich (INDIA) (purity; $\geq 99.99 \%$ (GC). The esterifying catalyst Boron Trifloride and solvent heptane were also purchased from Sigma (Sigma -Aldrich, India).

\section{Fat extraction}

Approximately $10 \mathrm{~g}$ of homogenized sample was weighed and transferred in the thimble of the Soxhlet apparatus. Lipids were extracted with $100 \mathrm{ml}$ of petroleum ether (60-80 B.P.) and two cycles for extraction were run to ensure complete extraction of fat. Total time required was approximately $3 \mathrm{hrs}$. However, for samples like gulabjamun, complete fat extraction was done by acid treatment and then ether extraction was done (AOAC, 2001) as fat was present in a bound state and could not be extracted completely by simple ether extraction.

After extraction, the mixture was dried with sodium thiosulfate $\left(\mathrm{Na}_{2} \mathrm{~S}_{2} \mathrm{O}_{3}\right)$ and filtered through filter paper. The 
solvent was removed by evaporation in a hot air oven at $80 \pm 5^{\circ} \mathrm{C}$. Total fat percent was calculated by weighing the soxhlet beaker.

\section{Sample preparation}

Approximately $0.2 \mathrm{~g}$ of oil sample was transferred to the flask, $10 \mathrm{ml}$ of $1.0 \mathrm{~N}$ methanolic $\mathrm{NaOH}$ was added and a known concentration of Internal Standard was added to the flask, which was then refluxed for $10 \mathrm{~min}$. About $5 \mathrm{ml}$ of $14 \%$ methanolic boron trifluoride $\left(\mathrm{BF}_{3}\right)$ $\mathrm{MeOH}$ ) was added and refluxed for an additional 2 min. About $5 \mathrm{ml} \mathrm{n}$-heptane was added to the flask through the condenser and then allowed to cool. The organic layer was then separated with centrifugation after adding $10 \mathrm{ml}$ concentrated $\mathrm{NaCl}$ solution. About $1.0 \mathrm{ml}$ of the top layer was transferred into a $10-\mathrm{ml}$ stoppered glass tube using a transfer pipette, and then the sample was diluted to the mark $(10 \mathrm{ml})$ with $\mathrm{n}$-heptane (AOAC, 1990).

\section{Gas chromatograph analysis of FAME}

FAMEs were analyzed using a GC Clarus 500 Chromatograph (Perkin Elmer, India) equipped with a fused silica capillary column SP-2560 (with column length- 30 $\mathrm{m}$ and internal diameter $320 \mu \mathrm{m}$ ) and flame ionization detector [FID]. High-purity nitrogen (99.999 \%) was used as the carrier gas with a set flow rate of $1 \mathrm{ml} / \mathrm{min}$ and hydrogen and zero air was used as fuel gas with flow rates $45 \mathrm{ml} / \mathrm{min}$ and $450 \mathrm{ml} / \mathrm{min}$ respectively. The oven temperature program was as follows: $4 \mathrm{~min}$ at 130 ${ }^{\circ} \mathrm{C}$, increased/ramped by $2.5^{\circ} \mathrm{C} / \mathrm{min}$ up to $240{ }^{\circ} \mathrm{C}$, and then further ramped at the rate of $5.0^{\circ} \mathrm{C}$ up to $260^{\circ} \mathrm{C}$ held for $20 \mathrm{~min}$. The injector and detector temperatures were 220 and $280{ }^{\circ} \mathrm{C}$, respectively. The injection volume was $2 \mu \mathrm{l}$ in split less mode (Modified AOAC, 2002). For Determination of Peroxide value of oil/fat IS: 548-Part 1, 1964 reaffirmed 2010 method (IS, 2010a) was used, while for Free fatty acid [FFA] analysis of oil /fat method used was IS: 548-Part 1, 1964 reaffirmed 2010 method (IS, 2010b).

\section{RESULTS AND DISCUSSION}

The total fat content of baked foods (cakes, pastry, pizza), confectionary (chocolates and cookies), snacks (potato fries, chips and aloo bhujia), indigenous snacks ( samosa, pakora and mathri) and indigenous sweets (jalebi, gulab jamun and laddoo) were analyzed. From the results it was found that highest fat content was present in chocolates ranging from $17-56 \%$, followed by chips and bhujia samples $12-25 \%$ and then fries 8$29 \%$. Fat content in pastry, cakes and pizza ranged from $7-12 \%, 11-23 \%$ and $10-15 \%$ respectively. Among indigenous snacks, fat content in samosa, pakora and mathri ranged from $12-25 \%, 10-21 \%$ and $13-17 \%$ respectively. In indigenous sweets, total fat content ranged from $9.8-17 \%, 7.8-12 \%$ and $10.5-15.5 \%$ respectively for jalebi, gulab jamun and laddoo samples. Results obtained for trans fatty acids were compared by categorizing the samples into branded and nonbranded (obtained from local vendors) in order to analyze the difference. The total fat and TFA compositions of analyzed vanaspati, margarines, cookies, pastry, biscuits, samosa, pakora, gulab jamun, jalebi etc. were given in Table 1. A large variation has been found in the trans fatty acid content among these food samples. The results also showed that TFA content in branded samples was significantly less than the non-branded samples with the mean value of TFA in branded and non-branded food groups as 1.781 and 6.125 respectively and the significant difference of 0.852 between the two groups (Fig. 1). The trans fat content in nonbranded vanaspati samples ranged from 18-23\% (which is beyond the limit regulated by FSSAI) (FDA, 2009) and $9.1 \%$ in the branded sample.

In margarine and butter samples trans fat \% in branded and non-branded samples ranged from $0.7-1.9 \%$ and $2.1-2.5 \%$ respectively. Among selected bakery products, trans fatty acid content in pastry/cakes ranged from $0.72-1.67 \%$ and $1.37-5.84 \%$ respectively for branded and non-branded samples. Trans fat content in pizza samples was found to be $0.8-1.2 \%$ for branded and $1.5-2.3 \%$ for non-branded. In the confectionaries, chocolate sample analyzed for TFA showed a very high range of trans fat in them with $32 \%$ and $26 \%$ trans fat in some of the non-branded samples, however, in branded chocolates trans fat was found to be $1.5-2.7 \%$. In cookies also. TFA was found to be high up to 3.4$5.8 \%$ in non-branded samples while in branded cookies trans fatty acid content ranged from $0.71-2.1 \%$. In this category nan- khatai samples were found to have the highest trans fat content. Such a high level of trans fats are basically because there are various unregulated small scale industries in India that manufacture biscuits and cookies using low grade and cheap fats. Among snacks, TFA content of potato fries ranged from 0.6 -

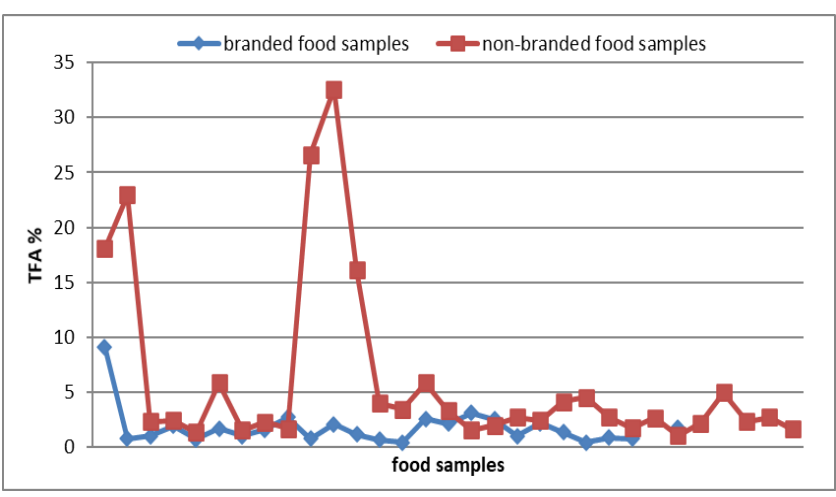

Fig. 1. Statistical plot for trans fats in branded and nonbranded food samples t-test showing a significant difference between branded and non-branded samples ( $t=$ $0.852>0.05$ ) . 
Table 1. Fatty acid profiling of selected food samples from Allahabad.

\begin{tabular}{|c|c|c|c|c|c|c|c|c|}
\hline $\begin{array}{l}\text { Sample } \\
\text { name }\end{array}$ & Sample no. & Fat \% & $\begin{array}{l}\text { Trans fat \% in } \\
\text { product }\end{array}$ & $\begin{array}{l}\text { Linoleic } \\
\text { acid methyl } \\
\text { ester }(\%)\end{array}$ & $\begin{array}{l}\text { Linolenic } \\
\text { acid me- } \\
\text { thyl ester } \\
(\%)\end{array}$ & $\begin{array}{l}\text { Elaidic } \\
\text { acid me- } \\
\text { thyl ester } \\
(\%)\end{array}$ & $\begin{array}{l}\text { Vaccenic } \\
\text { acid me- } \\
\text { thyl ester } \\
(\%)\end{array}$ & $\begin{array}{l}\text { Palmite- } \\
\text { laidic } \\
\text { acid me- } \\
\text { thyl ester } \\
(\%)\end{array}$ \\
\hline \multirow{3}{*}{ Vanaspati } & $\begin{array}{l}\text { *Sample } 1 \\
\text { (branded) }\end{array}$ & $100 \pm 0.1$ & $9.15 \pm 1.22$ & $5.53 \pm 0.31$ & $2.66 \pm 0.12$ & $0.65 \pm 0.08$ & $0.12 \pm 0.02$ & $2.51 \pm 0.58$ \\
\hline & $\begin{array}{l}\text { Sample } 2 \\
\text { (non-branded) }\end{array}$ & $100 \pm 0.5$ & $18.05 \pm 1.65$ & $5.78 \pm 0.97$ & $3.85 \pm 0.78$ & $1.68 \pm 0.43$ & $0.89 \pm 0.28$ & $5.85 \pm 2.92$ \\
\hline & $\begin{array}{l}\text { Sample } 3 \\
\text { (non-branded) }\end{array}$ & $100 \pm 0.5$ & $22.98 \pm 1.65$ & $4.68 \pm 0.99$ & $2.98 \pm 0.67$ & $0.64 \pm 0.24$ & $0.14 \pm 0.06$ & $15.08 \pm 1.07$ \\
\hline \multirow{3}{*}{ Margarine } & $\begin{array}{l}\text { Sample } 1 \\
\text { (non-branded) }\end{array}$ & $81.9 \pm 1.2$ & $2.336 \pm 0.33$ & $0.97 \pm 0.14$ & $0.67 \pm 0.08$ & $0.58 \pm 0.08$ & ND. & $0.11 \pm 0.06$ \\
\hline & $\begin{array}{l}\text { *Sample } 2 \\
\text { (branded) }\end{array}$ & $81 \pm 0.8$ & $1.008 \pm 0.23$ & $0.64 \pm 0.11$ & $0.19 \pm 0.08$ & $.23 \pm 0.10$ & ND. & $0.03 \pm 0.01$ \\
\hline & $\begin{array}{l}\text { *Sample } 3 \\
\text { (branded) }\end{array}$ & $80 \pm 0.6$ & $0.772 \pm 0.21$ & $0.55 \pm 0.04$ & $0.16 \pm 0.07$ & $0.05 \pm 0.01$ & ND. & $0.01 \pm 0.01$ \\
\hline Butter & $\begin{array}{l}\text { *Sample } 1 \\
\text { (branded) }\end{array}$ & $80 \pm 0.6$ & $1.934 \pm 0.27$ & $0.97 \pm 0.21$ & $0.42 \pm 0.09$ & $0.08 \pm 0.02$ & $0.18 \pm 0.07$ & $0.28 \pm 0.11$ \\
\hline \multicolumn{9}{|l|}{ Bakery } \\
\hline \multirow{3}{*}{ Pastry } & $\begin{array}{l}\text { Sample } 1 \\
\text { (branded) }\end{array}$ & $7.13 \pm 0.83$ & $0.726 \pm 0.17$ & $0.41 \pm 0.04$ & $0.28 \pm 0.05$ & $0.02 \pm 0.01$ & ND. & $0.01 \pm 0.01$ \\
\hline & $\begin{array}{l}\text { Sample } 2 \\
\text { (non-branded) }\end{array}$ & $11.92 \pm 0.95$ & $2.443 \pm 0.32$ & $0.88 \pm 0.12$ & $0.46 \pm 0.06$ & $0.21 \pm 0.04$ & $0.03 \pm 0.01$ & $0.86 \pm 0.14$ \\
\hline & $\begin{array}{l}\text { Sample } 3 \\
\text { (non-branded) }\end{array}$ & $8.26 \pm 0.62$ & $1.377 \pm 0.25$ & $0.54 \pm 0.07$ & $0.15 \pm 0.04$ & $0.03 \pm 0.02$ & ND. & $0.65 \pm 0.09$ \\
\hline \multirow{2}{*}{ Cake } & $\begin{array}{l}\text { Sample } 1 \\
\text { (non-branded) }\end{array}$ & $22.57 \pm 1.42$ & $5.845 \pm 0.68$ & $1.58 \pm 0.24$ & $1.68 \pm 0.54$ & $1.03 \pm 0.15$ & $0.27 \pm 0.02$ & $1.28 \pm 0.42$ \\
\hline & $\begin{array}{l}\text { *Sample } 2 \\
\text { (branded) }\end{array}$ & $11.34 \pm 0.96$ & $1.673 \pm 0.23$ & $0.69 \pm 0.07$ & $0.34 \pm 0.08$ & $0.18 \pm 0.05$ & $0.03 \pm 0.02$ & $0.43 \pm 0.02$ \\
\hline \multirow{4}{*}{ Pizza } & $\begin{array}{l}\text { Sample } 1 \\
\text { (non-branded) }\end{array}$ & $14.95 \pm 1.06$ & $1.553 \pm 0.33$ & $0.83 \pm 0.23$ & $0.25 \pm 0.06$ & $0.32 \pm 0.07$ & $0.07 \pm 0.03$ & $0.08 \pm 0.03$ \\
\hline & $\begin{array}{l}\text { Sample } 2 \\
\text { (non-branded) }\end{array}$ & $12.59 \pm 1.14$ & $2.294 \pm 0.42$ & $0.99 \pm 0.08$ & $0.56 \pm 0.05$ & $0.25 \pm 0.03$ & $0.21 \pm 0.01$ & $0.28 \pm 0.07$ \\
\hline & $\begin{array}{l}\text { Sample } 3 \\
\text { (non-branded) }\end{array}$ & $15.36 \pm 1.14$ & $1.69 \pm 0.34$ & $0.67 \pm 0.05$ & $0.26 \pm 0.06$ & $0.12 \pm 0.03$ & $0.31 \pm 0.04$ & $0.33 \pm 0.04$ \\
\hline & $\begin{array}{l}\text { Sample } 4 \\
\text { (branded) }\end{array}$ & $9.87 \pm 0.86$ & $1.014 \pm 0.27$ & $0.64 \pm 0.08$ & $0.21 \pm 0.06$ & $0.12 \pm 0.07$ & $0.07 \pm 0.03$ & ND. \\
\hline \multicolumn{9}{|c|}{ Confectionary } \\
\hline \multirow{5}{*}{$\begin{array}{l}\text { Choco- } \\
\text { lates }\end{array}$} & $\begin{array}{l}\text { Sample } 1 \\
\text { (non-branded) }\end{array}$ & $52.68 \pm 1.98$ & $26.643 \pm 2.82$ & $7.92 \pm 1.05$ & $7.82 \pm 1.12$ & $1.34 \pm 0.65$ & $0.05 \pm 0.02$ & $9.56 \pm 1.15$ \\
\hline & $\begin{array}{l}\text { Sample } 2 \\
\text { (non-branded) }\end{array}$ & $46.74 \pm 1.81$ & $32.585 \pm 1.18$ & $10.42 \pm 2.02$ & $8.35 \pm 1.47$ & $2.28 \pm 0.74$ & $0.87 \pm 0.05$ & $10.58 \pm 2.01$ \\
\hline & $\begin{array}{l}\text { Sample } 3 \\
\text { (non-branded) }\end{array}$ & $38.84 \pm 1.21$ & $16.118 \pm 1.68$ & $4.38 \pm 0.83$ & $7.24 \pm 0.96$ & $0.54 \pm 0.05$ & $0.12 \pm 0.02$ & $3.83 \pm 0.94$ \\
\hline & $\begin{array}{l}\text { *Sample } 4 \\
\text { (branded) }\end{array}$ & $28.2 \pm 0.93$ & $1.593 \pm 0.43$ & $0.85 \pm 0.21$ & $0.43 \pm 0.14$ & $0.21 \pm 0.05$ & $0.04 \pm 0.01$ & $0.32 \pm 0.08$ \\
\hline & $\begin{array}{l}\text { Sample } 5 \\
\text { (branded) }\end{array}$ & $17.5 \pm 0.96$ & $2.74 \pm 0.22$ & $1.23 \pm 0.24$ & $0.66 \pm 0.06$ & $0.25 \pm 0.07$ & $0.07 \pm 0.04$ & $0.54 \pm 0.06$ \\
\hline \multirow{5}{*}{ Cookies } & $\begin{array}{l}\text { Sample } 1 \\
\text { (non-branded) }\end{array}$ & $19.2 \pm 1.28$ & $3.98 \pm 0.63$ & $1.31 \pm 0.32$ & $1.28 \pm 0.27$ & $0.43 \pm 0.04$ & ND. & $0.96 \pm 0.14$ \\
\hline & $\begin{array}{l}\text { Sample } 2 \\
\text { (non-branded) }\end{array}$ & $18.15 \pm 0.96$ & $3.461 \pm 0.31$ & $1.18 \pm 0.43$ & $1.07 \pm 0.15$ & $0.37 \pm 0.07$ & $0.06 \pm 0.04$ & $0.79 \pm 0.12$ \\
\hline & $\begin{array}{l}\text { Sample } 3 \\
\text { (non-branded) }\end{array}$ & $13.7 \pm 0.75$ & $5.827 \pm 0.65$ & $1.67 \pm 0.12$ & $1.73 \pm 0.15$ & $0.99 \pm 0.07$ & $0.11 \pm 0.04$ & $1.38 \pm 0.11$ \\
\hline & $\begin{array}{l}\text { *Sample } 4 \\
\text { (branded) }\end{array}$ & $5.94 \pm 0.74$ & $0.747 \pm 0.12$ & $0.41 \pm 0.06$ & $0.12 \pm 0.02$ & $0.09 \pm 0.03$ & ND. & $0.12 \pm 0.04$ \\
\hline & $\begin{array}{l}{ }^{*} \text { Sample } 5 \\
\text { (branded) }\end{array}$ & $15.28 \pm 1.21$ & $2.109 \pm 0.37$ & $1.16 \pm 0.13$ & $0.63 \pm 0.06$ & $0.21 \pm 0.04$ & $0.03 \pm 0.02$ & $0.07 \pm 0.01$ \\
\hline
\end{tabular}


Tripathi, P. et al. / J. Appl. \& Nat. Sci. 13(1): 16 - 25 (2021)

\begin{tabular}{|c|c|c|c|c|c|c|c|c|}
\hline \multicolumn{9}{|l|}{ Snacks } \\
\hline \multirow{5}{*}{ Fries } & $\begin{array}{l}\text { Sample } 1 \\
\text { (non-branded) }\end{array}$ & $20.94 \pm 1.55$ & $3.323 \pm 0.48$ & $0.98 \pm 0.07$ & $0.84 \pm 0.13$ & $0.64 \pm 0.98$ & $0.18 \pm 0.07$ & $0.68 \pm 0.15$ \\
\hline & $\begin{array}{l}\text { Sample } 2 \\
\text { (non-branded) }\end{array}$ & $28.86 \pm 1.84$ & $1.551 \pm 0.24$ & $0.66 \pm 0.13$ & $0.42 \pm 0.08$ & $0.15 \pm 0.02$ & ND. & $0.32 \pm 0.06$ \\
\hline & $\begin{array}{l}\text { Sample } 3 \\
\text { (non-branded) }\end{array}$ & $19.15 \pm 1.43$ & $1.974 \pm 0.32$ & $1.12 \pm 0.16$ & $0.56 \pm 0.08$ & $0.11 \pm 0.03$ & $0.06 \pm 0.02$ & $0.13 \pm 0.06$ \\
\hline & $\begin{array}{l}\text { Sample } 4 \\
\text { (branded) }\end{array}$ & $21.53 \pm 1.48$ & $1.181 \pm 0.29$ & $0.78 \pm 0.17$ & $0.26 \pm 0.11$ & $0.12 \pm 0.06$ & $0.02 \pm 0.05$ & ND. \\
\hline & $\begin{array}{l}\text { Sample } 5 \\
\text { (branded) }\end{array}$ & $7.74 \pm 0.89$ & $0.661 \pm 0.27$ & $0.33 \pm 0.06$ & $0.27 \pm 0.06$ & $0.08 \pm 0.03$ & $0 . .4 \pm 0.02$ & ND. \\
\hline \multirow{2}{*}{ Chips } & $\begin{array}{l}\text { *Sample } 1 \\
\text { (branded) }\end{array}$ & $15.39 \pm 1.12$ & $0.445 \pm 0.12$ & $0.26 \pm 0.02$ & $0.12 \pm 0.06$ & $0.06 \pm 0.02$ & ND. & ND. \\
\hline & $\begin{array}{l}\text { Sample } 2 \\
\text { (non-branded) }\end{array}$ & $18.67 \pm 1.49$ & $2.724 \pm 0.35$ & $1.03 \pm 0.15$ & $0.53 \pm 0.07$ & $0.23 \pm 0.04$ & $0.07 \pm 0.02$ & $0.86 \pm 0.12$ \\
\hline \multirow{3}{*}{ Aloo Bhujia } & $\begin{array}{l}\text { Sample } 1 \\
\text { (non-branded) }\end{array}$ & $21.96 \pm 0.76$ & $2.444 \pm 0.18$ & $1.21 \pm 0.35$ & $0.47 \pm 0.12$ & $0.27 \pm 0.06$ & $0.02 \pm 0.01$ & $0.47 \pm 0.08$ \\
\hline & $\begin{array}{l}\text { Sample } 2 \\
\text { (branded) }\end{array}$ & $29.59 \pm 2.18$ & $2.591 \pm 0.39$ & $1.49 \pm 0.17$ & $0.75 \pm 0.08$ & $0.11 \pm 0.05$ & ND. & $0.24 \pm 0.08$ \\
\hline & $\begin{array}{l}\text { *Sample } 3 \\
\text { (branded) }\end{array}$ & $19.67 \pm 1.42$ & $2.124 \pm 0.37$ & $1.18 \pm 0.21$ & $0.65 \pm 0.09$ & $0.21 \pm 0.07$ & N.D. & $0.12 \pm 0.06$ \\
\hline \multicolumn{9}{|c|}{ Indigenous snacks } \\
\hline \multirow{4}{*}{ Samosa } & $\begin{array}{l}\text { Sample } 1 \\
\text { (branded) }\end{array}$ & $14.67 \pm 1.32$ & $3.110 \pm 0.21$ & $0.96 \pm 0.21$ & $0.78 \pm 0.21$ & $0.11 \pm 0.06$ & ND. & $1.27 \pm 0.61$ \\
\hline & $\begin{array}{l}\text { Sample } 2 \\
\text { (non-branded) }\end{array}$ & $15.52 \pm 1.52$ & $4.125 \pm 0.77$ & $1.13 \pm 0.08$ & $1.48 \pm 0.32$ & $0.36 \pm 0.07$ & $0.01 \pm 0.01$ & $1.14 \pm 0.08$ \\
\hline & $\begin{array}{l}\text { Sample } 3 \\
\text { (non-branded) }\end{array}$ & $12.35 \pm 0.97$ & $4.534 \pm 0.41$ & $1.25 \pm 0.37$ & $1.33 \pm 0.32$ & $0.64 \pm 0.13$ & ND. & $1.37 \pm 0.43$ \\
\hline & $\begin{array}{l}\text { Sample } 4 \\
\text { (branded) }\end{array}$ & $24.67 \pm 1.96$ & $2.542 \pm 0.22$ & $1.29 \pm 0.12$ & $0.48 \pm 0.04$ & $0.34 \pm 0.02$ & $0.04 \pm 0.01$ & $0.43 \pm 0.07$ \\
\hline \multirow{4}{*}{ Pakora } & $\begin{array}{l}\text { Sample } 1 \\
\text { (non-branded) }\end{array}$ & $20.33 \pm 2.01$ & $2.714 \pm 0.31$ & $0.82 \pm 0.11$ & $0.66 \pm 0.09$ & $0.21 \pm 0.07$ & $0.06 \pm 0.04$ & $0.96 \pm 0.16$ \\
\hline & $\begin{array}{l}\text { Sample } 2 \\
\text { (non-branded) }\end{array}$ & $16.76 \pm 1.55$ & $1.727 \pm 0.36$ & $0.53 \pm 0.12$ & $0.28 \pm 0.06$ & $0.13 \pm 0.03$ & ND. & $0.78 \pm 0.14$ \\
\hline & $\begin{array}{l}\text { Sample } 3 \\
\text { (non-branded) }\end{array}$ & $17.29 \pm 1.09$ & $2.656 \pm 0.32$ & $0.76 \pm 0.11$ & $0.31 \pm 0.06$ & $0.79 \pm 0.07$ & ND. & $0.79 \pm 0.16$ \\
\hline & $\begin{array}{l}\text { Sample } 4 \\
\text { (non-branded) }\end{array}$ & $9.85 \pm 0.98$ & $1.081 \pm 0.27$ & $0.64 \pm 0.07$ & $0.32 \pm 0.06$ & $0.09 \pm 0.03$ & ND. & $0.03 \pm 0.02$ \\
\hline \multirow{4}{*}{ Mathri } & $\begin{array}{l}\text { Sample } 1 \\
\text { (branded) }\end{array}$ & $15.83 \pm 1.15$ & $1.02 \pm 0.30$ & $0.61 \pm 0.08$ & $0.25 \pm 0.06$ & $0.10 \pm 0.04$ & ND. & $0.05 \pm 0.02$ \\
\hline & $\begin{array}{l}\text { Sample } 2 \\
\text { (non-branded) }\end{array}$ & $13.63 \pm 0.83$ & $2.17 \pm 0.24$ & $0.65 \pm 0.09$ & $0.40 \pm 0.06$ & $0.12 \pm 0.02$ & ND. & ND. \\
\hline & $\begin{array}{l}\text { Sample } 3 \\
\text { (non-branded) }\end{array}$ & $16.94 \pm 1.20$ & $4.96 \pm 0.63$ & $2.31 \pm 0.61$ & $0.87 \pm 0.98$ & $0.11 \pm 0.06$ & ND. & $1.62 \pm 0.18$ \\
\hline & $\begin{array}{l}\text { Sample } 4 \\
\text { (non-branded) }\end{array}$ & $13.28 \pm 0.97$ & $2.33 \pm 0.47$ & $1.13 \pm 0.11$ & $0.39 \pm 0.07$ & $0.12 \pm 0.04$ & ND. & $0.68 \pm 0.09$ \\
\hline \multicolumn{9}{|c|}{ Indigenous sweets } \\
\hline \multirow{4}{*}{ Jalebi } & $\begin{array}{l}\text { Sample } 1 \\
\text { (non-branded) }\end{array}$ & $10.62 \pm 1.07$ & $2.725 \pm 0.45$ & $1.12 \pm 0.40$ & $0.83 \pm 0.21$ & $0.06 \pm 0.02$ & $0.03 \pm 0.01$ & $0.75 \pm 0.15$ \\
\hline & $\begin{array}{l}\text { Sample } 2 \\
\text { (non-branded) }\end{array}$ & $10.1 \pm 1.18$ & $1.669 \pm 0.35$ & $0.93 \pm 0.21$ & $0.42 \pm 0.16$ & $0.12 \pm 0.04$ & $0.07 \pm 0.04$ & $0.13 \pm 0.08$ \\
\hline & $\begin{array}{l}\text { Sample } 3 \\
\text { (branded) }\end{array}$ & $9.82 \pm 1.01$ & $2.173 \pm 0.41$ & $1.07 \pm 0.87$ & $0.61 \pm 0.03$ & $0.14 \pm 0.06$ & $0.03 \pm 0.02$ & $0.65 \pm 0.13$ \\
\hline & $\begin{array}{l}\text { Sample } 4 \\
\text { (non-branded) }\end{array}$ & $16.45 \pm 1.52$ & $2.426 \pm 0.37$ & $1.13 \pm 0.74$ & $0.84 \pm 0.32$ & $0.17 \pm 0.06$ & $0.07 \pm 0.02$ & $0.33 \pm 0.06$ \\
\hline \multirow{5}{*}{$\begin{array}{l}\text { Gulab } \\
\text { jamun }\end{array}$} & $\begin{array}{l}\text { Sample } 1 \\
\text { (non-branded) }\end{array}$ & $10.52 \pm 0.86$ & $2.177 \pm 0.32$ & $0.74 \pm 0.05$ & $0.72 \pm 0.08$ & $0.18 \pm 0.03$ & $0.33 \pm 0.07$ & $0.21 \pm 0.02$ \\
\hline & $\begin{array}{l}\text { Sample } 2 \\
\text { (non-branded) }\end{array}$ & $11.32 \pm 1.21$ & $3.314 \pm 0.84$ & $1.08 \pm 0.16$ & $0.63 \pm 0.08$ & $0.16 \pm 0.03$ & $0.21 \pm 0.04$ & $1.23 \pm 0.08$ \\
\hline & $\begin{array}{l}\text { *Sample } 3 \\
\text { (branded) }\end{array}$ & $11.48 \pm 0.97$ & $1.378 \pm 0.27$ & $0.78 \pm 0.08$ & $0.23 \pm 0.05$ & $0.08 \pm 0.02$ & $0.22 \pm 0.06$ & $0.06 \pm 0.04$ \\
\hline & $\begin{array}{l}\text { Sample } 4 \\
\text { (branded) }\end{array}$ & $7.87 \pm 0.99$ & $0.424 \pm 0.13$ & $0.21 \pm 0.05$ & $0.06 \pm 0.02$ & $0.01 \pm 0.01$ & $0.14 \pm 0.05$ & ND. \\
\hline & $\begin{array}{l}\text { Sample } 5 \\
\text { (non-branded) }\end{array}$ & $11.69 \pm 1.12$ & $2.792 \pm 0.44$ & $1.04 \pm 0.13$ & $0.78 \pm 0.08$ & $0.53 \pm 0.05$ & $0.31 \pm 0.02$ & $0.13 \pm 0.02$ \\
\hline \multirow{4}{*}{ Laddoo } & $\begin{array}{l}\text { Sample } 1 \\
\text { (branded) }\end{array}$ & $12.57 \pm 1.09$ & $0.858 \pm 0.12$ & $0.59 \pm 0.09$ & $0.13 \pm 0.06$ & $0.01 \pm 0.01$ & $0.11 \pm 0.04$ & $0.02 \pm 0.01$ \\
\hline & $\begin{array}{l}\text { Sample } 2 \\
\text { (branded) }\end{array}$ & $14.24 \pm 1.20$ & $0.788 \pm 0.15$ & $0.61 \pm 0.06$ & $0.15 \pm 0.03$ & ND. & ND. & $0.03 \pm 0.01$ \\
\hline & $\begin{array}{l}\text { Sample } 3 \\
\text { (non-branded) }\end{array}$ & $10.72 \pm 0.81$ & $3.182 \pm 0.47$ & $1.08 \pm 0.21$ & $0.38 \pm 0.05$ & $0.09 \pm 0.02$ & ND. & $1.63 \pm 0.14$ \\
\hline & $\begin{array}{l}\text { Sample } 4 \\
\text { (non-branded) }\end{array}$ & $15.17 \pm 0.96$ & $2.183 \pm 0.38$ & $1.16 \pm 0.13$ & $0.75 \pm 0.10$ & $0.21 \pm 0.04$ & $0.08 \pm 0.02$ & ND. \\
\hline
\end{tabular}


$1.1 \%$ and $1.5-3.3 \%$ for branded and non-branded samples. Aloo bhujia has less variability in TFA content ranging from $2.1 \%$ to $2.6 \%$ for branded as well as nonbranded samples. Among Indigenous foods, trans fat $\%$ of branded and non-branded samples were found to be $2.5-3.1 \%$ and $4.1-4.6 \%$ respectively for samosa, 0.78 $-1.2 \%$ and $2.1-5.0 \%$ respectively for mathri. Pakora samples analyzed were all non-branded and the trans fat content in them were found to be $1.0-2.7 \%$. The high level of trans fat in these samples may be due to high trans containing vanaspati that are used for frying. In the same way the samples of gulab jamun, jalebi and laddoo have their trans fat \% in branded and nonbranded sample ranged from $0.4-1.4 \%$ and $2.1-3.4 \%$ respectively for gulab jamun, $1.7-2.1 \%$ and $1.7-2.8 \%$ respectively for jalebi and $0.78-0.85 \%$ and $2.1-3.2 \%$ for laddoo respectively. The range of trans fats observed in different food categories is given in Table 2. Since it was not feasible to print the GC graph for all the 64 samples, hence GC chromatogram of one sample from each food group have been given in Fig. 3 .

Among the various categories, the highest trans content was found in chocolates and then cookies, followed by cakes/pastry and then potato fries. Pizza samples were found to have comparatively less trans fat. Among Indian traditional foods, samosa is found to contain maximum trans fatty acids followed by Gulabjamun. Pakora samples were found to have the least trans fat content. Of the five TFA isomers characterized, the most commonly identified were Elaidic Acid (C18:1), Palmitelaidic Acid (C16:1), and Linolenaidic Acid (C18:2).

Analysis of various food samples indicated that general concentrations of TFA in analyzed food samples are high with approximately $8 \%$ of samples having more than $5 \%$ TFA whereas $58 \%$ of samples were found to have more than $2 \%$ TFA (as \% total composition). Only $4.5 \%$ of the samples were found to contain TFA less than $0.5 \%$. (can be marked to be trans fat-free) (Fig. 2). It was also observed that among the food items studied, only $17 \%$ samples (10 out of 60 ) were labelled for trans fatty acid content, all the other samples were unlabelled and consumers are totally ignorant about the ingredients and quality of these products. The street food and snacks vendors comprise of an unorganized sector who are mostly unaware of the regulations to check trans fatty acid content in food. The public at large do not have an opportunity to make informed choices which help in reducing TFA intake as well as a decrease in health risk. The present study showed the significance of studying all similar products and point out the need for laying regulation on all such products. Different amount of TFA was found in the same food item sourced from different outlets such as a branded sweet shop, local sweet shop and roadside vendors. A reason for this variability is that products often contain

\section{trans fat in market}

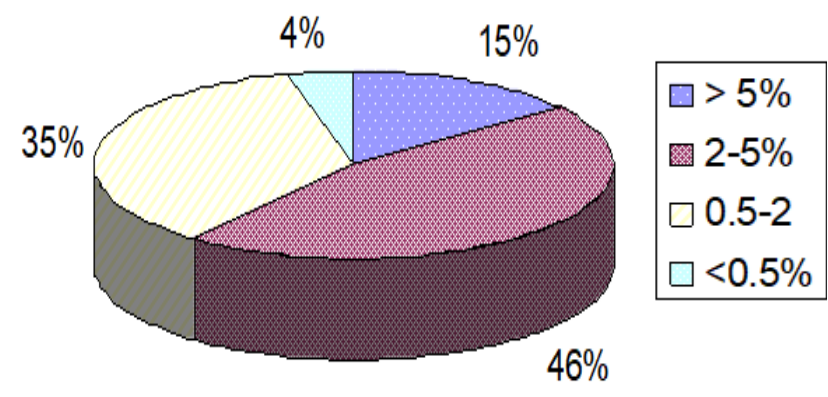

Fig. 2. Distribution of trans fatty acid in all the selected food samples analyzed for trans fatty acids.

a blend of partially hydrogenated vegetable oils (PHVO) of different sources such as soybean, canola, palm oil, corn and sunflower oil. The proportions of hydrogenated and non-hydrogenated oils in these foods are also varied to obtain the desired physical properties Similar variability was observed in studies done in Australia and Canada, where a high level of TFA was found in over $10 \%$ of food items analyzed and the authors also found great variability in TFA content in crackers, doughnuts and chocolate wafer biscuits (Wagner et al., 2008). Earlier studies have also reported varied TFA level within similar food items like cookies, fries, doughnuts, nuggets, pizza etc. due to type of oil used in the preparation (McCarthy et al., 2008, Trattner et al., 2015). This variability reflects the difficulty in assessing TFA intake in a population, especially by dietary methods which depend on food composition tables.

The Trans fatty acids reported in this study for Laddoo and Mathri samples were found to be lesser (0.78-3.2 and $0.78-5.0$ respectively) than the earlier reported study (6.2-7.3 and 2.16-8.2 respectively); however, the

Table 2. Summary of the level of Trans fatty acids identified in selected food groups.

\begin{tabular}{lll}
\hline Product Type & $\begin{array}{l}\text { Branded (trans } \\
\text { fat \%) }\end{array}$ & $\begin{array}{l}\text { Non-branded } \\
\text { (trans fat\%) }\end{array}$ \\
\hline $\begin{array}{l}\text { Margarine/ } \\
\text { butter }\end{array}$ & $0.7-1.9 \%$ & $2.1-2.5 \%$ \\
$\begin{array}{l}\text { Toffees/ } \\
\text { chocolates }\end{array}$ & $1.5-2.7 \%$ & $16.1-32.6 \%$ \\
Cookies & $0.71-2.1 \%$ & $3.4-5.8 \%$ \\
Cake/Pastry & $0.72-1.67 \%$ & $1.37-5.84 \%$ \\
Samosa & $2.5-3.1 \%$ & $4.1-4.6 \%$ \\
Pakora & No sample & $1.0-2.7 \%$ \\
Jalebi & $1.7-2.1 \%$ & $1.7-2.7 \%$ \\
Gulabjamun & $0.4-1.4 \%$ & $2.1-3.4 \%$ \\
Fries & $0.6-1.1 \%$ & $1.5-3.3 \%$ \\
Pizza & $0.8-1.2 \%$ & $1.5-2.3 \%$ \\
Snacks & $0.3-2.6 \%$ & $2.4-2.8 \%$ \\
Mathri & $0.78-1.2 \%$ & $2.1-5.0 \%$ \\
Laddoo & $0.78-0.85 \%$ & $2.1-3.2 \%$ \\
\hline
\end{tabular}


Tripathi, P. et al. / J. Appl. \& Nat. Sci. 13(1): 16 - 25 (2021)
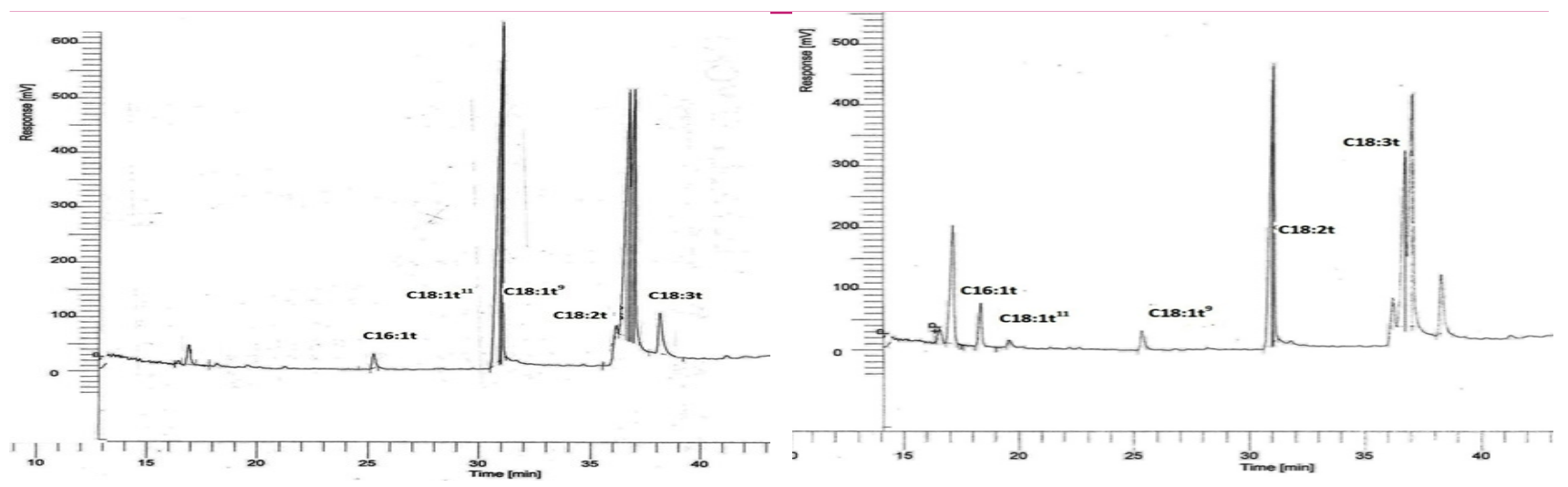

a. GC chromatogram of Clarified butter.

b. GC chromatogram of Cookies.
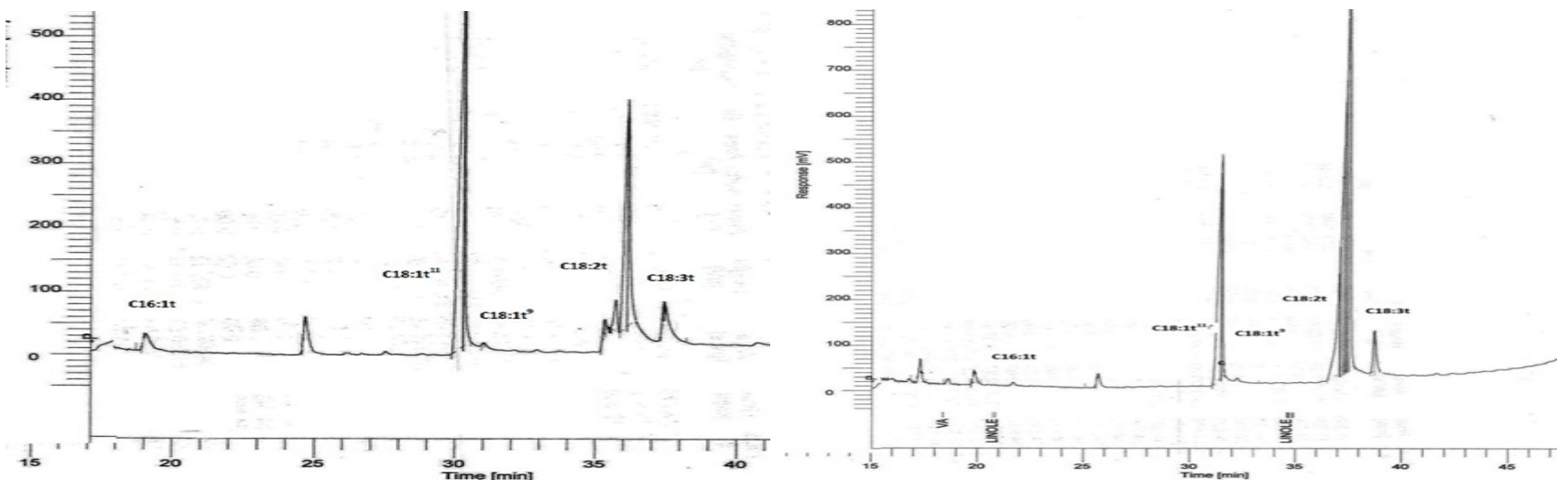

c. GC chromatogram of Jalebi

d. GC chromatogram of Cake
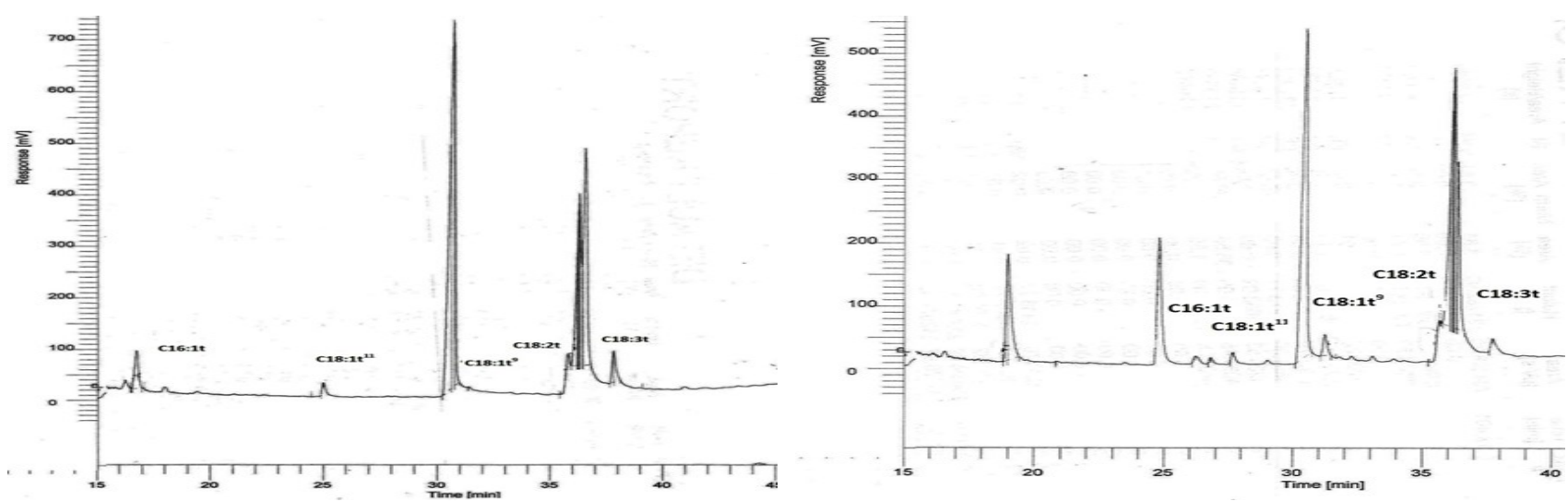

e. GC chromatogram of Mathri

f. GC chromatogram of Chocolate
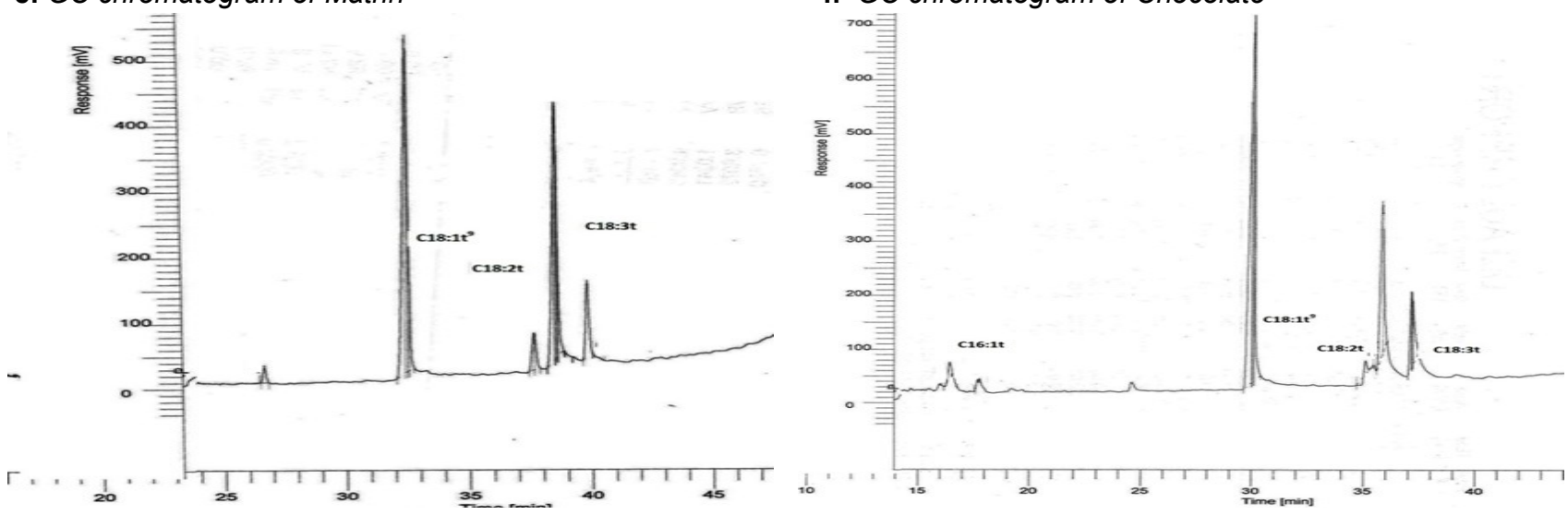

g. GC chromatogram of Pizza

h. GC chromatogram of Laddoo

Fig. 3 (a-h). GC chromatogram of one sample of each food group samples. 
Samosa samples were found to have higher TFA (2.54.6) than earlier reported study (0.21-3.6) (Karn et al., 2013). In another recent study, the level of TFA reported in chips samples were found to be ranging from 0.055-0.14 (Joshee et al., 2019) which was to be similar with the data reported for branded chips samples in this study. For samples like Pastry, Toffees, Jalebi, Gulab jamun, French fries and Pizza, no data has been reported from India earlier.

A number of approaches have been initiated by governmental and public health organizations in different countries to reduce the amount of trans-fatty acid (TFA) intakes. In India also there is need to initiate such programs to bring down the trans fat content in products from Indian food market like various other countries have reduced the trans fat in their food by strict regulations (Bysted et al., 2009, L'Abbe et al., 2009). There is an urgent need to make public aware of ill effects of TFA, because India is home to the highest number of atherosclerosis in the world (Katan, 2006) and consumption of trans fatty acid in diet further increases the risk of atherosclerosis and other diseases like obesity, coronary heart diseases, prostate cancer, breast can- cer, etc. National Institute of Nutrition carried out a risk analysis of trans fats in the Indian Diet and stated that not more than $1 \%$ of total energy should be derived from TFA (FDA, 2009) if the risk of cardiovascular disease has to be kept at a safe level. As per the draft notifications of FSSAl, the limit of TFA in fats/oils should not be more than $3 \%$ by weight on and from $1^{\text {st }}$ January 2021 and should not be more than $2 \%$ by weight on and from $1^{\text {st }}$ January 2022. It also mentions that to safeguard the health of consumers, trans fat should be eliminated from Indian foods as soon as possible (FSSAI 2019). However, in the results reported in this study, it was found that most of the food samples sold in the market have high trans content and even more major issue is that these foods items are generally more popular in small children. Hence it is important for population and individual health that TFAs should be removed from the food supply and should be replaced by cis-unsaturated fats from vegetable oils rather than saturated fats from tropical oils and animal fats. The database provided in this report also focuses on the need to regulate the trans fat content in nonbranded and unlabelled food products.

Table 3. Quality parameters of oil/fat used in selected food samples (Branded and Non-branded).

\begin{tabular}{|c|c|c|c|c|c|}
\hline Branded Sample & $\begin{array}{l}\text { Peroxide value } \\
(\mathrm{Meq} / 100 \mathrm{~g})\end{array}$ & $\begin{array}{l}\text { Free fatty acid } \\
(\%)\end{array}$ & $\begin{array}{l}\text { Non-branded } \\
\text { Samples }\end{array}$ & $\begin{array}{l}\text { Peroxide value } \\
\text { (Meq/100g) }\end{array}$ & $\begin{array}{l}\text { Free fatty acid } \\
(\%)\end{array}$ \\
\hline Vanaspati 1 & $0.081 \pm .034$ & $0.18 \pm 0.08$ & Vanaspati 1 & $0.162 \pm .065$ & $0.18 \pm 0.07$ \\
\hline Margarine 3 & $0.193 \pm .082$ & $0.25 \pm 0.09$ & Vanaspati 2 & $0.184 \pm .074$ & $0.30 \pm 0.08$ \\
\hline Butter 1 & $0.743 \pm .037$ & $0.41 \pm 0.10$ & Margarine1 & $0.233 \pm .082$ & $0.41 \pm 0.09$ \\
\hline Samosa 1 & $0.401 \pm .019$ & $0.63 \pm 0.07$ & Margarine 2 & $0.421 \pm .029$ & $0.63 \pm 0.12$ \\
\hline Samosa 2 & $0.175 \pm .021$ & $0.92 \pm 0.21$ & Samosa 1 & $0.238 \pm .023$ & $0.88 \pm 0.17$ \\
\hline Pakora 1 & $0.045 \pm .008$ & $0.35 \pm 0.17$ & Samosa 2 & $0.401 \pm .019$ & $0.35 \pm 0.04$ \\
\hline Jalebi1 & $0.349 \pm .071$ & $0.51 \pm 0.11$ & Samosa 3 & $0.546 \pm .029$ & $0.46 \pm 0.04$ \\
\hline Jalebi 2 & $0.149 \pm .031$ & $0.71 \pm 0.14$ & Pakora 1 & $0.428 \pm .017$ & $0.71 \pm 0.13$ \\
\hline Gulabjamun 1 & $0.148 \pm .018$ & $0.94 \pm 0.09$ & Pakora 2 & $0.329 \pm .028$ & $0.81 \pm 0.21$ \\
\hline Gulabjamun2 & $0.233 \pm .039$ & $1.21 \pm 0.43$ & Pakora 3 & $0.286 \pm .073$ & $1.15 \pm 0.26$ \\
\hline Gulabjamun 3 & $0.204 \pm .041$ & $0.18 \pm 0.06$ & Jalebi 1 & $0.447 \pm .039$ & $0.29 \pm 0.03$ \\
\hline Chocolate 1 & $0.179 \pm .029$ & $0.19 \pm 0.05$ & Jalebi 2 & $0.529 \pm .087$ & $0.41 \pm 0.03$ \\
\hline Chocolate 2 & $0.231 \pm .048$ & $0.19 \pm 0.09$ & Gulab jamun 1 & $0.262 \pm .019$ & $0.81 \pm 0.16$ \\
\hline Cookies 1 & $0.088 \pm .003$ & $0.22 \pm 0.14$ & Gulabjamun 2 & $0.270 \pm .095$ & $1.13 \pm 0.18$ \\
\hline Cookies 2 & $0.125 \pm .039$ & $0.35 \pm 0.21$ & Chocolate 1 & $0.193 \pm .049$ & $1.32 \pm 0.23$ \\
\hline Pastry 1 & $0.212 \pm .041$ & $0.3 \pm 0.11$ & Chocolate 2 & $0.164 \pm .017$ & $0.21 \pm 0.09$ \\
\hline Pastry 2 & $0.297 \pm .056$ & $0.34 \pm 0.17$ & Chocolate 3 & $0.218 \pm .043$ & $0.38 \pm 0.05$ \\
\hline Cake 1 & $0.075 \pm .008$ & $0.36 \pm 0.15$ & Cookies 1 & $0.237 \pm .038$ & $0.68 \pm 0.12$ \\
\hline Fries 1 & $0.051 \pm .008$ & $0.29 \pm 0.12$ & Cookies 2 & $0.350 \pm .014$ & $0.77 \pm 0.05$ \\
\hline Fries 2 & $0.186 \pm .039$ & $0.32 \pm 0.08$ & Pastry 1 & $0.323 \pm .092$ & $0.83 \pm 0.15$ \\
\hline Fries3 & $0.129 \pm .021$ & $0.3 \pm 0.09$ & Pastry 2 & $0.314 \pm .086$ & $1.02 \pm 0.32$ \\
\hline Fries 4 & $0.174 \pm .048$ & $0.35 \pm 0.05$ & Fries 1 & $0.299 \pm .087$ & $1.25 \pm 0.13$ \\
\hline Pizza 1 & $0.272 \pm .076$ & $0.5 \pm 0.06$ & Pizza 1 & $0.389 \pm .099$ & $1.62 \pm 0.21$ \\
\hline Pizza 2 & $0.196 \pm .062$ & $0.45 \pm 0.05$ & Pizza2 & $0.296 \pm .079$ & $2.21 \pm 0.28$ \\
\hline Chips & $0.329 \pm .081$ & $0.5 \pm 0.09$ & Aloo Bhujia 1 & $0.472 \pm .092$ & $2.53 \pm 0.43$ \\
\hline Aloo 1 & $0.241 \pm .064$ & $0.52 \pm 0.14$ & Aloo Bhujia 2 & $0.295 \pm .058$ & $0.69 \pm 0.12$ \\
\hline Laddoo 1 & $0.258 \pm .073$ & $0.65 \pm 0.21$ & Laddoo 1 & $0.139 \pm .022$ & $0.72 \pm 0.08$ \\
\hline Laddoo 2 & $0.196 \pm .038$ & $0.7 \pm 0.11$ & Laddoo 2 & $0.058 \pm .007$ & $0.7 \pm 0.09$ \\
\hline Mathri 1 & $0.213 \pm .019$ & $0.74 \pm 0.17$ & Mathri1 & $0.213 \pm .019$ & $0.75 \pm 0.05$ \\
\hline Mathri 2 & $0.165 \pm .017$ & $0.74 \pm 0.15$ & Mathri 2 & $0.244 \pm .058$ & $1.62 \pm 0.21$ \\
\hline
\end{tabular}


Tripathi, P. et al. / J. Appl. \& Nat. Sci. 13(1): 16 - 25 (2021)

\begin{abstract}
Analysis of peroxide value and free fatty acid content in food samples

The results of quality parameters like Free fatty acids and Peroxide Value of fat samples that were extracted from the selected food items are detailed in Table 3 . It was observed that none of the food samples exceeded the maximum limit of peroxide value for oils/fats (maximum permissible limit of peroxide value in oil/fats is specified to be $10 \mathrm{mEq} / \mathrm{Kg}$ of oil, as per FSSAI regulation). Results obtained for the free fatty acid content in various branded and non-branded food samples showed that among the branded samples 11 out 30 samples were found to contain free fatty acid more than $0.5 \%$, whereas in non-branded samples 21 out of 30 samples contains more than $0.5 \%$ FFA, which is the maximum permissible limit for free fatty acids specified in Food Safety and Standards Regulations (FSSR, 2010). Higher FFA value causes high acidity of the oils which was caused by the breakdown of fat after storage or use.
\end{abstract}

\section{Conclusion}

The present study concluded that most of the food samples sold in the major market places from Allahabad have high trans content which may lead to health issues like cardiovascular disease, obesity, coronary heart diseases, prostrate cancer, breast cancer, etc. and even more major issue is that these foods items generally target and are more popular in small children. Hence it is important for population and individual health that TFAs should be removed from the food supply and should be replaced by cis-unsaturated fats from vegetable oils rather than saturated fats from tropical oils and animal fats. Food Industry may require a suitable time frame for adopting healthier trans fat alternatives. The Indian health department should also take such initiatives. When regulations are emphasizing on labelling the TFA content on the food products, arrays of unlabelled products were found which are high in trans fat content and are required to be strictly regulated. Hence there is a need for strong food regulations to bring levels of trans fats in processed foods to negligible levels.

\section{ACKNOWLEDGEMENTS}

I Prashasti Tripathi, would like to thank University Grants Commission (UGC), New Delhi for providing Junior research fellowship for conducting this research (JRF).

\section{Conflict of interest}

The authors declare that they have no conflict of interest.

\section{REFERENCES}

1. Aggrawal, A., Gupta, R., Varma, K. \& Mathur, B. (2008). High trans fatty acid content in common Indian fast foods. Nutrition and Food Science, 38(6), 564-569.

2. AOAC (1990). AOAC Fatty Acid in Oils and Fats Preparation of Methyl Ester Boron Trifluoride Method, 15th Edition, Association of Official Analytical Chemists Official Method 969.33, AOAC International, Washington DC, 400 -405 .

3. AOAC (2001) AOAC Fat (Total, Saturated and Unsaturated) in Foods, Hydrolytic Extraction Gas Chromatographic Method, 18th Edition, Association of Official Analytical Chemists Official Method 996.06. AOAC International, Arlington.

4. AOAC (2002). AOAC official method Fat (total, saturated, unsaturated, and monosaturated) in cereal products: acid hydrolysis capillary gas chromatographic. Association of Official Analytical Chemists (AOAC) official methods of analysis. 17th ed.Method 996.01,

5. Bysted, A., Mikkelsen, A. E. \& Leth T. (2009). Substitution of trans fatty acids in foods on the Danish market. Europian Journal of Lipid Science and Technology, 111, 574-83.

6. Codex (1985). Codex Alimentarius, Guidelines on nutrition labelling, CAC/GL Retreived from www.codexaliment arius.org/input/download/standards/34/CX G_002e.pdf .

7. EFSA (2004). Opinion of the Scientific Panel on Dietetic Products, Nutrition and Allergies on a Request from the Commission Related to the Presence of Trans Fatty Acids in Foods and the Effect on Human Health of the Consumption of Trans Fatty Acids. The EFSA Journal, 81, 149 .

8. FDA (2009). Food labeling: Trans fatty acids in nutrition labeling, nutrient content claims, and health claims. Fed. Regist., 68, 41434- 41506,

9. FSSR (2010). Food product standards (Part 5.2), Fats, oils and fat emulsions, Food Safety and Standards Regulation, 279-280.

10. FSSAI (2019). Use of trans fat free Claim-reg. Food Safety and Standards Authority of India. F.No 1-94/FSSAI/SP (Claims and Advertisement)/2017-Pt., notification dated $22^{\text {nd }}$ July 2019.

11. Hosomi K., Kiomo H. \& Kunisawa J., (2020). Fatty acid metabolism in the host and commensal bacteria for the control of intestinal immune responses and diseases, Gut Microbes, 11(3), 276-284.

12. IS (2010a). Indian standards (IS) 548 (1964 reaffirmed 2010) - methods of sampling and tests for oils and fats, Part 1,sampling, physical and chemical tests, determination of acid value in fats and oils, 29-31.

13. IS (2010b). Indian standards (IS) 548 (1964 reaffirmed 2010) - methods of sampling and tests for oils and fats, Part 1 ,sampling, physical and chemical tests, determination of peroxide value in fats and oils, 63-65.

14. Joshee K, Abhang T. \& Kulkarni R. (2019). Fatty acid profiling of 75 Indian snack samples highlights overall low trans fatty acid content with high polyunsaturated fatty acid content in some samples, PloS ONE, 14(12), e0225798. https://doi. org/10.1371/journal.pone.022 5798

15. Karn S., Abraham R.A. \& Ramakrishnan L. (2013). Assessment of trans fatty acid content in widely consumed 
snacks by Gas Chromatography in a Developing Country. Journal of Food and Nutrition Sciences, 4, 1281-1286.

16. Katan, B. M. (2006). Regulation of trans fats: The gap, the Polder and McDonald's French fries. Atherosclerosis Supplement, 7, 63-66.

17. Kodali, D.R, (2005). Trans Fats - Chemistry, Occurrence, Functional Need in Foods and Potential Solutions. In Kodali, D.R. and List, G.R. (eds.) Trans Fats Alternatives, AOCS Press, Champaign Illinois, United States, 1-25.

18. L'Abbe, M. R., Stender, S., Skeaff, M., Ghafoorunissa, R., \& Tavella, M (2009). Approaches to removing trans fats from the food supply in industrialized and developing countries. European Journal of Clinical Nutrition, 63, S50S67.

19. McCarthy J., Barr D. \& Sinclair A. (2008). Determination of Trans Fatty Acid Levels by FTIR in Processed Foods in Australia. Asia Pacific Journal of Clinical Nutrition, 17(3), 391-396.

20. McNaughton S.A, Hughes M.C. \& Marks G.C. (2007). Validation of a FFQ to estimate the intake of PUFA using plasma phospholipid fatty acids and weighed food records. British Journal of Nutrition, 97, 561-568.

21. Micha, R. \& Mozaffarian, D (2009). Trans fatty acids: effect on metabolic syndrome, heart disease and diabetes. Nature Reviews Endocrinology, 5(6), 335-344.

22. Mozaffarian, D., Cao, H., King, I. B., Lemaitre, R. N., Song, X., Siscovick, D. S. \& Hotamisligil, G. S., (2010). Trans-Palmitoleic Acid, Metabolic Risk Factors, and NewOnset Diabetes in U.S. Adults. Annals of Internal Medicine, 153, 790-799.

23. Trattner S. Becker W, Wretling S., Ohrvik V, Mattisson I. (2015). Fatty acid composition of Swedish bakery products, with emphasis on trans-fatty acids. Journal of Food Chemistry 175, 423-430.

24. Wagner, K. H., Plasser, E., Proell, C., \& Kanzler, S. (2008) Comprehensive studies on the trans fatty acid content of Austrian foods: Convenience products, fast food and fats. Food Chemistry, 108, 1054-1060.

25. WHO (2003). Diet, Nutrition and the Prevention of Chronic Diseases: World Health Organization, \& Food and Agriculture Organization (WHO/FAO) Technical Report Series, 916, 0-160

26. Zhu Y., Bo Y. \& Liu Y. (2019). Dietary total fat, fatty acids intake and risk of cardiovascular disease: a dose response meta analysis of cohort studies. Journal of Lipids in Health and Disease, 18, 91. 